\title{
The impact of hallux valgus on foot kinematics: A cross-sectional, comparative study
}

\author{
Kevin Deschamps ${ }^{\text {a,b,d,*}, ~ I v a n ~ B i r c h ~}{ }^{c}$, Kaat Desloovere ${ }^{\mathrm{d}}$, Giovanni A. Matricali ${ }^{\mathrm{e}}$ \\ ${ }^{a}$ Division of Musculoskeletal Disorders, University Hospitals Leuven, KU-Leuven, Belgium \\ ${ }^{\mathrm{b}}$ Department of Rehabilitation Sciences (KU-Leuven) - Research Center for Musculoskeletal, Rehabilitation, Belgium \\ ${ }^{\mathrm{c}}$ Faculty of Health, Division of Podiatry, University of Brighton, Robert Dodd Building, Darley Road, Eastbourne, East Sussex BN20 7UR, UK \\ ${ }^{\mathrm{d}}$ Laboratory for Clinical Motion Analysis, University Hospital Pellenberg, KU-Leuven, Weligerveld 1, 3212 Pellenberg, Belgium \\ ${ }^{\text {e }}$ Division of Musculoskeletal Disorders, University Hospitals Leuven, KU-Leuven, Weligerveld 1, 3212 Pellenberg, Belgium
}

\section{A R T I C L E I N F O}

Article history:

Received 20 August 2009

Received in revised form 22 March 2010

Accepted 28 March 2010

\section{Keywords:}

Kinematics

Gait analysis

Foot joints

Hallux valgus

Multi-segment foot model

\begin{abstract}
A B S T R A C T
Background: Hallux valgus is a very common foot deformity in modern societies. The impact of this condition on foot function has been described qualitatively and quantitatively. Published pathomechanical models are mainly underpinned by findings originating from plantar pressure measurements. However, the kinematical patterns of the many foot segments during gait have not been quantified. This study aims to evaluate the kinematics of the various foot segments in the presence of this deformity.

Methods: Using the Oxford Foot Model and a 12-camera Motion Analysis System, gait analysis was conducted on a convenience sample of 20 participants with hallux valgus and compared to that of 22 randomly selected symptom-free volunteers. Differences between temporal and kinematical data between groups were analyzed using the unpaired parametric Student $t$-test (significance level $p<0.01$ ). Results: During specific gait events, a different range of motion was found at several inter-segment angles. Particularly, the range of motion of the hallux (sagittal plane) and hindfoot (frontal-transverse planes) during stance were significantly different $(p<0.01)$.

Conclusion: Sagittal plane kinematics of the hallux is affected by the first ray deformity in this condition. However, the impact on other segments was found to be limited. This suggests that the patho-mechanical consequences remain limited to the weight bearing function of the first ray.
\end{abstract}

(c) 2010 Elsevier B.V. All rights reserved.

\section{Introduction}

One of the most common deformities encountered in podiatric and orthopaedic practice is hallux valgus (HV) [1]. Commonly referred to as a bunion deformity, HV is characterized by the progressive subluxation and valgus angulation of the first metatarsophalangeal joint (1MTPJ) in combination with pronation of the proximal phalanx [2-4]. Genetic predisposition, inappropriate shoe wear, trauma and biomechanical compensation for structural and functional deformities (hindfoot eversion) have been reported as contributors to this disorder [3,5]. Shoe adaptations, custom made insoles and digital silicone orthoses have been reported as conservative treatment options [6,7].

\footnotetext{
* Corresponding author at: Division of Musculoskeletal Disorders, University Hospital-Leuven, KU-Leuven, Weligerveld 1, 3212 Pellenberg, Belgium. Tel.: +32 16338024

E-mail address: kevin.deschamps@uz.kuleuven.ac.be (K. Deschamps).
}

Surgical correction is the more invasive treatment option, with more than 100 different techniques having been described $[8,9]$.

An important step in the management of foot pathologies is the objective analysis of foot mechanics in dynamic conditions. Several authors have demonstrated that patients with HV have an altered plantar pressure pattern in the forefoot compared to asymptomatic individuals [3,10-12]. These studies have shown that the most commonly observed patho-mechanical manifestation is the first ray insufficiency during mid-stance and propulsion, which causes an increased load over the central metatarsal heads [3,13]. Postoperative outcome has also been evaluated by means of pedobarography $[14,15]$.

Much less is known about the kinematic behavior of the foot affected by HV. Kinematic analysis of the foot has been challenging for many years, partially because of technological limitations and also due to the intrinsic complexity of the foot [16]. However, over the last decades several research groups have been able to develop three-dimensional multi-segment foot models (3-DMFM) [16-20]. This approach is thought to enhance the biomechanical understanding of the foot as it allows measuring the kinematical 
behavior of specific segments of the foot [16,21-23]. In order to obtain objective information on foot kinematics in patients with $\mathrm{HV}$, a cross-sectional comparative study was undertaken. We hypothesized that inter-segmental range of motion measured in a HV population would differ from that measured in a healthy control group.

\section{Methods}

\subsection{Participants}

Two study groups were recruited over a 3-month period. A convenience sample of adults with hallux valgus (HV, $N=20)$ was voluntarily recruited through the institutions' Foot \& Ankle Department. Potential participants with a HV angle $>25^{\circ}$, good peripheral pulses and adequate sensation (Semmes-Weinstein monofilament $10 \mathrm{~g}$ ) were recruited. The decision to select patients with a $\mathrm{HV}$ angle $>25^{\circ}$ was based on a commonly used clinical grading system of HV deformity [24]. Taking into account the physiological deviation between the first metatarsal and phalanx patients with a HV angle $>25^{\circ}$ are considered to have at least a mild deformity.

Normative data were gathered from a group of symptom-free adult volunteers (control group (CG), $N=22$ ), recruited through advertisements at the same hospital for a period of 3 months. From the total of 46 persons who responded spontaneously to the advertisement, a random sample of 22 participants was selected using a random sample table. For both groups, potential participants were excluded from the study if they had a history of trauma of the foot or lower limbs, a foot deformity such as pes cavus, pes planus, metatarsus adductus, claw toes (screened through standard clinical examination) or a systemic or neurological disorder. The first intermetatarsal angle and the hallux valgus angle of the HV group were measured with the Picture Archiving and Communication System of the hospital. This radiographic assessment was only possible for the HV group because standard radiographs were not available for the randomly selected CG. Hallux valgus angle in the control group was evaluated qualitatively through clinical examination, as this was shown to have excellent inter-observer repeatability [24,25]. Following clinical examination, all subjects in the control group had 'no deformity'. The local Ethics Committee granted approval for the study and informed consent was obtained from all subjects. Participant demographics are summarized in Table 1.

\subsection{Gait analysis}

A $10 \mathrm{~m}$ walkway with two strain-gauge forces plates integrated in the floor was used to record the walking trials of the participants. This walkway allowed using the midgait protocol which ensures a natural gait [26].

Three-dimensional foot and lower limb motion data of both groups were measured using a 612 Vicon Motion Capture System (Oxford Metrics, Oxford, United Kingdom). Force plate data (Advanced Mechanical Technology Inc., Watertown, MA) were used to accurately determine the temporal parameters of the gait cycle. Force plate data were sampled at $100 \mathrm{~Hz}$.

\subsection{Foot kinematics}

The Oxford Foot model (OFM) described by Stebbins et al. [20] was implemented in order to quantify the foot kinematics of both groups. Passive reflective markers

\section{Table 1}

Demographical and temporal parameters of both groups (control vs. hallux valgus, significance level at $p<0.05$ ).

\begin{tabular}{|c|c|c|c|}
\hline Characteristics & Control & Hallux valgus & $p$-Value \\
\hline \multicolumn{4}{|l|}{ Demographics } \\
\hline Number of participants & 22 & 20 & - \\
\hline Age (average + range) & $\begin{array}{l}37.5 \\
\text { (20-60 years) }\end{array}$ & $\begin{array}{l}47.4 \\
\text { (18-65 years) }\end{array}$ & - \\
\hline Sex (males/females) & $9 / 13$ & $4 / 16$ & - \\
\hline Affected side (right/left) & - & $19 / 17$ & - \\
\hline $\mathrm{BMI}^{\mathrm{a}}$ (average + range) & $\begin{array}{l}23.1 \\
(18.3-28.1 \%)\end{array}$ & $\begin{array}{l}25.1 \\
(17.2-34.1 \%)\end{array}$ & 0.064 \\
\hline Investigated foot (right/left) & $11 / 11$ & $11 / 9$ & - \\
\hline $\mathrm{IMA}^{\circ}$ (average + range) & - & $\begin{array}{l}14.2 \\
\left(10.7-20.7^{\circ}\right)\end{array}$ & - \\
\hline HV angle $D$ (average + range) & - & $\begin{array}{l}33.6 \\
\left(25-48.4^{\circ}\right)\end{array}$ & - \\
\hline \multicolumn{4}{|l|}{ Temporal parameters } \\
\hline Gait cycle duration $(\mathrm{s})^{\mathrm{b}}$ & $1.14( \pm 0.08)$ & $1.13( \pm 0.09)$ & 0.984 \\
\hline Stance duration (\%) & $61.8( \pm 1.84)$ & $61.9( \pm 2.44)$ & 0.856 \\
\hline Swing duration (\%) & $38.2( \pm 1.84)$ & $38.1( \pm 2.44)$ & 0.856 \\
\hline
\end{tabular}

\footnotetext{
a Body mass index.

b Denotes average $\pm 1 \mathrm{SD}$. IMA ${ }^{\circ}$ : first intermetatarsal angle, HV angle $D=$ hallux valgus angle.
}

with a diameter of $14 \mathrm{~mm}$ were positioned at the anatomical landmarks as described by the authors of the model. Inter-segment angle calculations in the OFM are performed by adopting the joint coordinate system [27]

\subsection{Measurement protocol and data analysis}

Kinematic data were collected during a single test occasion. After the markers had been attached, a static trial was undertaken in order to establish the local segment reference frames. During this static trial, individuals were asked to stand in a relaxed position, with the knees extended and with the feet along the $X$-axis of the laboratory. The relative orientation of the leg, hindfoot and forefoot, recorded during this static trial, was subsequently captured and considered as the relative position. As a consequence, no off set was applied to the dynamic data. Subsequently, the measurements of the dynamic trials were undertaken, with individuals walking at a self-selected speed until 5 adequate walking trials were recorded for his/her dominant foot (determined as the foot used to initiate gait).

\subsection{Statistical analysis}

In order to explore any differences in the demographic and temporal parameters of both groups, the parametric Student $t$-test was used.

Temporal parameters of gait included gait cycle duration, stance duration and swing duration.

For the statistical analysis of kinematic data, two types of data were considered for the median trial of each patient $[17,28]$.

First, the relative motion occurring at each inter-segment angle for each subject was calculated, for the seven gait phases [29]: loading response $(0-10 \%)$, midstance (10-30\%), terminal stance (30-50\%), pre-swing (50-60\%), initial swing (60$73 \%$ ), mid-swing (73-87\%) and terminal swing (87-100\%). The relative motion for each gait phase was defined as the difference between the maximum and minimum angle during that phase. To adjust for multiple tests over the seven test points, a Bonferroni correction was used to achieve a 5\% overall error rate. Differences in kinematic data between groups was therefore considered to be significant at a level of $p<0.01$ using the parametric Student $t$-test.

Secondly, the mean inter-segmental angle was calculated for each group to represent the timing of the movement throughout the complete gait cycle. Only the inter-segment angles where a significant difference in relative motion was found will be presented in this article.

\section{Results}

No significant differences were found in the temporal parameters between groups (Table 1 ).

In the sagittal plane, the HV group demonstrated a significantly increased dorsiflexion motion $(p<0.01)$, whereas the averaged position of the hallux during stance was persistently higher in the CG (Fig. 1). Differences in motion also reached a significant level during terminal swing (Table 2).

In both groups, a gradual decrease of the forefoot-hindfoot plantarflexion angle was observed throughout the first $50 \%$ of gait (Fig. 2). Pre-swing was characterized by a distinct plantarflexion of the forefoot and reached a peak position just after toe-off. Only during mid-swing a significant difference was found in the motion, with more plantarflexion in the CG $(p<0.01)$. In the HV group, an increased range of adduction motion during mid-swing at the forefoot-hindfoot angle was observed $(p<0.01)$ (Table 2$)$.

During loading response a mean eversion of $5.3^{\circ}(\mathrm{CG})$ and $6.5^{\circ}$ (HV group) was observed at the hindfoot (Fig. 3). During midstance and terminal stance the hindfoot inverted gradually followed by eversion of the hindfoot during initial swing. During mid-stance, a decrease in inversion was observed in the HV Group $(p<0.01)$, whereas during pre-swing, a significantly increased hindfoot eversion was found in the HV group (Table 2).

External rotation at the hindfoot-tibia angle was observed during the first $40 \%$ of gait cycle followed by internal rotation throughout terminal stance and pre-swing (Fig. 3). During terminal stance, the HV group showed a restricted motion compared to the CG $(p<0.01)$.

A small period of plantarflexion followed by a dorsiflexion of the forefoot relatively to the tibia throughout mid-stance and terminal stance was observed in both groups (Fig. 1). Pre-swing was characterized by rapid plantarflexion, with a mean peak 

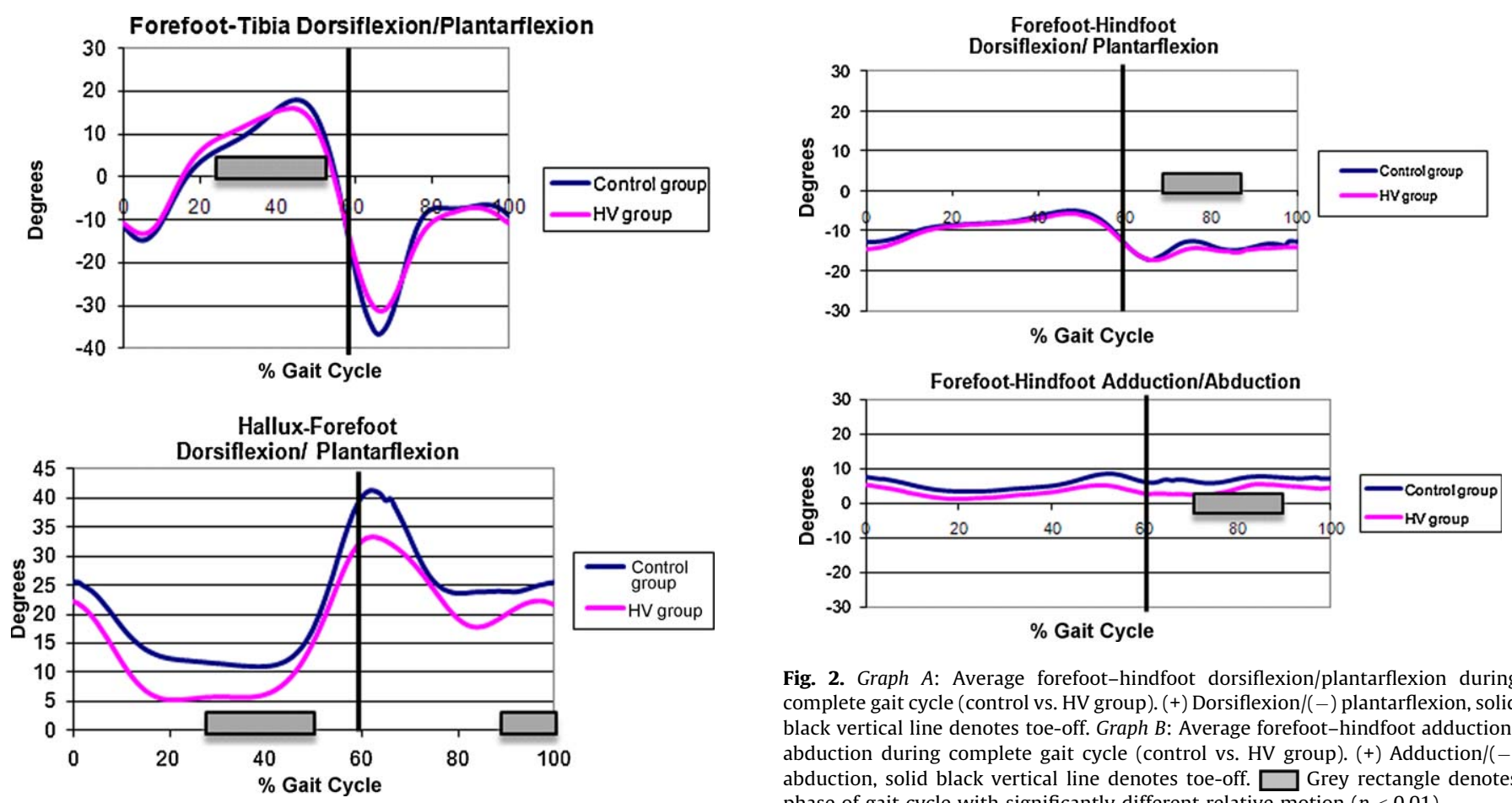

Fig. 2. Graph A: Average forefoot-hindfoot dorsiflexion/plantarflexion during complete gait cycle (control vs. HV group). (+) Dorsiflexion/(-) plantarflexion, solid black vertical line denotes toe-off. Graph $B$ : Average forefoot-hindfoot adduction/ abduction during complete gait cycle (control vs. HV group). (+) Adduction/(-) abduction, solid black vertical line denotes toe-off. $\square$ Grey rectangle denotes phase of gait cycle with significantly different relative motion $(p<0.01)$.

Fig. 1. Graph A: Average forefoot-tibia dorsiflexion/plantarflexion during complete gait cycle (control vs. HV group). (+) Dorsiflexion/(-) plantarflexion, solid black vertical line denotes toe-off. Graph B: Average hallux-forefoot dorsiflexion/ plantarflexion during complete gait cycle (control vs. HV group). (+) Dorsiflexion $/(-)$ plantarflexion, solid black vertical line denotes toe-off. Grey rectangle denotes phase of gait cycle with significantly different relative motion $(p<0.01)$.

plantarflexion position of $36.5^{\circ}$ (CG) and $31.2^{\circ}$ (HV group). During terminal stance, significantly decreased plantarflexion was observed in the HV group $(p<0.01)$.

\section{Discussion}

Many attempts have been made to describe foot function in patients affected with HV. Previous studies have mostly used pedobarographic technology to achieve this objective $[3,10,12]$. We investigated, for the first time, the kinematic behavior of the segments of the foot in subjects with HV and compared it with normative data (CG).

The demographic characteristics of both groups showed a considerable difference in the male/female ratio. Whereas in the CG a ratio of nearly 1:1 was observed, for the HV group this ratio was $1: 4$. The convenience sampling technique and the higher prevalence [3] of HV in women (1:8) have played a considerable role and therefore account for the different male/female ratio in this study.

The impact of foot pathology on temporal parameters of gait has been previously reported $[22,23]$. Increased stance duration,
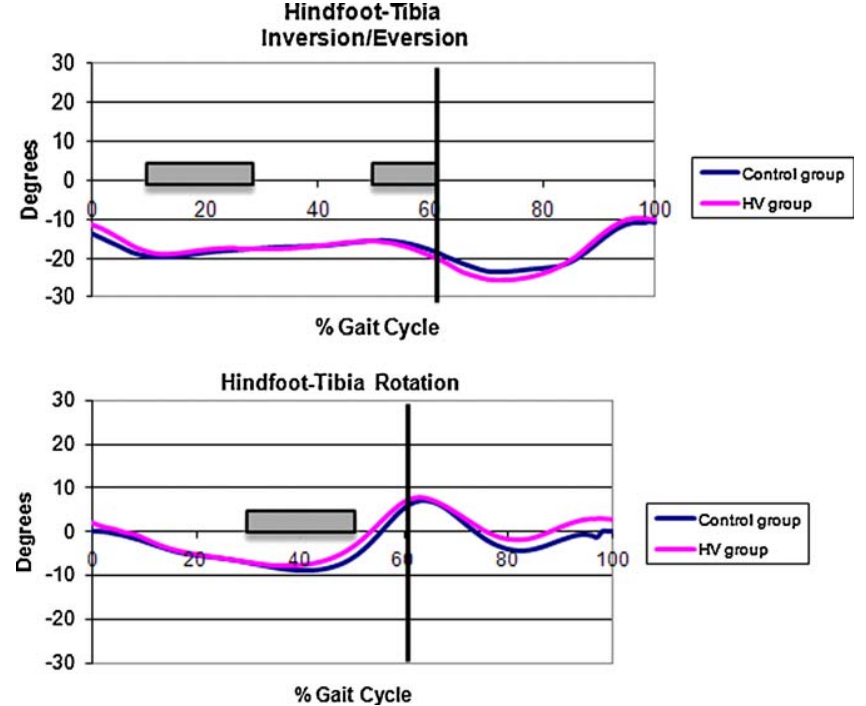

Fig. 3. Graph A Average hindfoot-tibia inversion/eversion during complete gait cycle (control vs. HV group). (+) Inversion/(-) eversion, solid black vertical line denotes toe-off. Graph $B$ : Average hindfoot-tibia internal/external rotation during complete gait cycle (control vs. HV group). (+) Internal/(-) external, solid black vertical line denotes toe-off. $\square$ Grey rectangle denotes phase of gait cycle with significantly different relative motion $(p<0.01)$.

Table 2

Motion at the inter-segment angles (mean \pm SD; degrees) showing significant differences between the control group and HV group.

\begin{tabular}{|c|c|c|c|c|}
\hline Inter-segment angle & Gait phase & Control group & HV group & $p$-Value \\
\hline Hallux-forefoot DF/PF & Terminal stance & $4.0 \pm 6.5$ & $7.9 \pm 6.6$ & $<0.01$ \\
\hline Hallux-forefoot DF/PF & Terminal swing & $-1.7 \pm 7.6$ & $3.7 \pm 7.8$ & $<0.01$ \\
\hline Forefoot-hindfoot DF/PF & Mid-swing & $-2.1 \pm 3.0$ & $0.3 \pm 3.7$ & $<0.01$ \\
\hline Forefoot-hindfoot $\mathrm{AD} / \mathrm{AB}$ & Mid-swing & $2.1 \pm 2.8$ & $3.9 \pm 3.4$ & $<0.01$ \\
\hline Hindfoot-tibia Inv/Ev & Mid-stance & $2.0 \pm 2.8$ & $-0.3 \pm 4.8$ & $<0.01$ \\
\hline Hindfoot-tibia Inv/Ev & Pre-swing & $-1.4 \pm 3.1$ & $-3.1 \pm 2.5$ & $<0.01$ \\
\hline Hindfoot-tibia Int./Ext. & Terminal stance & $3.6 \pm 2.0$ & $1.3 \pm 4.9$ & $<0.01$ \\
\hline Forefoot-tibia DF/PF & Terminal stance & $10.4 \pm 7.0$ & $4.1 \pm 8.5$ & $<0.01$ \\
\hline
\end{tabular}

DF: dorsiflexion, PF: plantarflexion, AD: adduction, AB: abduction, Inv: inversion, Ev: eversion, Int.: internal rotation, Ext.: external rotation. 
reduced walking speed, a decreased stride length and an increased cadence are common findings. Contrary to previous studies, we did not find significant alterations in the temporal parameters tested.

Motion patterns of individual foot segments were found to be similar in both groups, but in agreement with the previous literature. Some differences were observed in the hindfoot-tibia inversion/eversion and forefoot-hindfoot dorsiflexion/plantarflexion with the data provided by Stebbins et al. [20,30], however, these mainly reflected a general off set of the angle waveforms. In fact, the waveforms show a similar pattern and also a similar relative motion during the sub-phases of stance.

In the control group, the motion pattern of the hallux in the sagittal plane showed good face validity compared with data from other studies [19-21]. The best comparison was found with the data provided by Canseco et al. [21] and Simon and co-workers [9]. It should be stressed that the data published by Stebbins et al. [20] represented normative values of 15 healthy children between 6 and 14 years old. Therefore some reservation is required when these are compared to our population. Normative values for adult males and females recorded with the OFM have recently been presented at scientific meetings [30]. Canseco et al. [21] found a significant reduction of hallux dorsiflexion during pre-swing through mid-swing in a population affected with hallux rigidus. Whereas hallux rigidus is characterized by arthrosis of the 1MTPJ with progressive decrease of motion, hallux valgus may involve degenerative changes and loss of motion, but is primarily characterized by progressive subluxation of the 1MTPJ.

In our HV population we found an increased dorsiflexion motion at the hallux during terminal stance. A reasonable explanation for this difference can be the relatively decreased dorsiflexion angle present throughout the first $30 \%$ of the gait cycle. This is caused by the dorsiflexion/adduction at the first tarsometatarsal joint which is inherently present in hallux valgus, which results in a metatarsal head to be more cranially located compared to the other MTH and a first metatarsal joint to be closer to its neutral position $\left(0^{\circ}\right)$ instead of being relatively dorsiflexed. An increased dorsiflexion motion, probably caused by an earlier onset of the dorsiflexion movement would therefore be a consequence of the first ray malalignment. Additionally, it can be observed that the slope of the curve during pre-swing is more pronounced in the CG. This is a typical finding for an adequate push-off, were adequate plantarflexion of the first ray and dorsiflexion at the hallux occurs fluently [31]. However, in the HV group this pattern seems to be less pronounced, probably caused by a decreased lever arm for dorsiflexion movement.

The sagittal motion pattern at the ankle complex was similar between the groups and in accordance with previous publications [32]. The typical 'three rocker pattern' was observed and showed no significant differences.

Excessive subtalar joint eversion has been suggested as a causative factor for HV. The eversion should be seen as a consequence of a functional or structural deformity such as first ray hyper mobility, forefoot varus, forefoot supinatus and congenitally tight gastrocnemius causing equinus. Abnormal subtalar joint eversion unlocks the midtarsal joint and leads to kinematic deviations, both proximally and distally. The most affected periods are those of terminal stance and pre-swing were the vertical ground reaction force rises above resting body weight [32].

The OFM measures the relative motion of the calcaneus compared to the tibia. We found a significant difference $(p<0.01)$ in the relative motion during mid-stance and preswing. In both sub-phases of stance, the HV group showed increased eversion of the hindfoot, indicating a less stable foot. Despite these differences being only small, statistical significance was reached for these parameters. This provides quantitative evidence that hindfoot eversion is a causative factor for hallux valgus, as alluded above.
Internal rotation of the hindfoot during terminal stance was more prominent in the control group. This finding is related to the more pronounced inversion of the hindfoot and therefore the tendency of the hindfoot to regain a more 'neutral' alignment.

The forefoot-tibia dorsiflexion angle showed a similar pattern in both groups, but the peak positions (at $50 \%$ and $70 \%$ of gait cycle) were more pronounced in the CG. A reduced amount of dorsiflexion was observed during terminal stance in the hallux valgus group $(p<0.01)$. The flattening of peak angles has been attributed to antalgic gait in numerous publications [32]. Pain over the forefoot due to metatarsalgia, hallux valgus and skin callosities inhibits push-off which is often visualized in a butterfly diagram. Reduced elasticity of the plantar aponeurosis or mechanical dysfunction of the first ray may also be a causative factor for this difference in forefoot kinematics.

Despite the valuable findings, we have to acknowledge certain limitations and elements which are subject to some discussion in our study.

First, it may have been more appropriate to select a gendermatched control group as this would have avoided the different male/female ratio between the two groups. Additionally, such a design would have accounted for the recent observations that women have a more flexible foot compared to men [30]. More studies are needed in order to fully appreciate the effect of gender, body weight, age and speed on foot kinematics.

Accurately defining segmental kinematics requires accurate palpation of anatomical landmarks. Estimating the reliability of landmark identification is impeded by many factors and therefore not yet adequately determined. To overcome this dilemma, estimation of the intra-inter-observer and between-within day repeatability is often undertaken [17]. In some HV subjects, the medially located bursitis over the dislocated first metatarsal head prevented adequate palpation. The impact of this altered morphology onto the acquired kinematic data has not been investigated. Ideally, this should be undertaken for each pathology or deformity.

Another important factor which should be addressed is the reliability of tracking foot bones from skin markers and the role of soft tissue interposition [33]. Muscle contraction and inertial effects are two sources which may account for this artefact. However, it is generally accepted that greater motion artefact exists at the knee and hip due to the presence of the quadriceps complex [34]. Finally, the limited number of HV participants should be recognized. A convenience sample of $20 \mathrm{HV}$ subjects resulted in a moderate power for this study. Inclusion of additional patients with HV is currently in progress in order to draw further conclusions. Pre- and post-surgical assessment will be undertaken in order to quantify the impact of bony and soft tissue corrections on foot kinematics.

\section{Conclusion}

This study demonstrated that subjects with hallux valgus have a different inter-segmental range of motion in the affected foot during barefoot walking when compared to asymptomatic subjects. The significant differences observed in this study provide some evidence which supports previously suggested pathological mechanisms. However, these differences were small, which indicates that the impact of the first ray deformity remains limited to the hallux and that other segments of the foot are not affected in a major way.

\section{Conflict of interest statement}

The authors declare that no conflicts of interest exist. 


\section{References}

[1] Taranto J, Taranto MJ, Bryant AR, Singer KP. Analysis of dynamic angle of gait and radiographic features in subjects with hallux abducto valgus and hallux limitus. J Am Podiatr Med Assoc 2007;97(3):175-88.

[2] Jahss MH. Disorders of the foot and ankle. Philadelphia: WB Saunders; 1992 .

[3] Kernozek TW, Elfessi A, Sterriker S. Clinical and biomechanical risk factors of patients diagnosed with hallux valgus. J Am Podiatr Med Assoc 2003; 93(2):97-103.

[4] Okuda R, Kinoshita M, Yasuda T, Jotoku T, Kitano N, Shima H. The shape of the lateral edge of the first metatarsal head as a risk factor for recurrence of hallux valgus. J Bone Joint Surg Am 2007;89:2163-72.

[5] Schoenhaus HD, Cohen RS. Etiology of the bunion. J Foot Ankle Surg 1992;31:25.

[6] Nawoczenski DA, Epler ME. Orthotics in functional rehabilitation of the lower limb. Philadelphia: WB Saunders; 1997. pp. 116-155.

[7] Tang SF, Chen CP, Pan JL, Chen JL, Chau-Peng L, Ngok-Kiu C. The effects of a new foot-toe orthosis in treating painful hallux valgus. Arch Phys Med Rehabil 2002;83(12):1792-5.

[8] Hamilton RJ, Gray A, Kumar S. Surgical treatment of hallux valgus-the current practice in Scotland. The Foot 2005;15(2):65-7.

[9] Monga P, Kumar A, Simons A. Outcome following surgery for hallux valgus: the patients' perspective. Foot Ankle Surg 2006;12(2):95-8.

[10] Hutton WC, Dhanendran M. The mechanics of normal and hallux valgus feet-a quantitative study. Clin Orthop Relat Res 1981;157:7-13.

[11] Blomgren M, Turan I. Gait analysis in hallux valgus. J Foot Ankle Surg 1991;30(1):70-1.

[12] Waldecker U. Pedographic analysis of hallux valgus deformity. Foot Ankle Surg 2004;10(3):121-4

[13] Nyska M, Liberson A, McCabe C, Linge K, Klenerman L. Plantar foot pressure distribution in patients with hallux valgus treated by distal soft tissue procedure and proximal metatarsal osteotomy. Foot Ankle Surg 1998;4: 35-41.

[14] Saro C, Andrén B, Felländer-Tsai L, Lindgren U, Arndt A. Plantar pressure distribution and pain after distal osteotomy for hallux valgus: a prospective study of 22 patients with 12-month follow-up. The Foot 2007;17(2): 84-93.

[15] Dhukaram V, Hullin MG, Kumar CS. The Mitchell and scarf osteotomies for hallux valgus correction: a retrospective, comparative analysis using plantar pressures. J Foot Ankle Surg 2006;45(6):400-9.

[16] Baker R, Robb J. Foot models for clinical gait analysis. Gait Posture 2006;23:399-400.
[17] Carson MC, Harrington ME, Thompson N, O'Connor JJ, Theologis TN. Kinematic analysis of a multi-segment foot model for research and clinical applications: a repeatability analysis. J Biomech 2001;34:1299-307.

[18] MacWilliams BA, Cowley M, Nicholson DE. Foot kinematics during adolescent gait. Gait Posture 2003;17(3):214-24.

[19] Simon J, Doederlein L, McIntosh AS, Metaxiotis D, Bock HG, Wolf SI. The Heidelberg foot measurement method: development, description and assessment. Gait Posture 2006;23:411-24.

[20] Stebbins J, Harrington M, Thompson N, Zavatsky A, Theologis T. Repeatability of a model for measuring multi-segment foot kinematics in children. Gait Posture 2006;23:401-10.

[21] Canseco K, Long J, Marks R, Harris G. Quantitative motion analysis in hallux valgus: rehabilitative insight. Arch Phys Med Rehab 2007;88:E10.

[22] Canseco K, Long J, Marks R, Khazzam M, Harris G. Quantitative characterization of gait kinematics in patients with hallux rigidus using the Milwaukee Foot Model. J Orthop Res 2008;26(4):419-27.

[23] Ness ME, Long J, Marks R, Harris G. Foot and ankle kinematics in patients with posterior tibial tendon dysfunction. Gait Posture 2008;27:331-9.

[24] Garrow AP, Papageorgiou A, Silman AJ, Thomas E, Jayson MI, Macfarlane G] The grading of hallux valgus. The Manchester Scale. J Am Podiatr Med Assoc 2001;91(2):74-8.

[25] Menz HB, Munteanu SE. Radiographic validation of the Manchester scale for the classification of hallux valgus deformity. Rheumatology 2005:44:1061-6.

[26] Bus SA, de Lange A. A comparison of the 1-step, 2-step, and 3-step protocols for obtaining barefoot plantar pressure data in the diabetic neuropathic foot. Clin Biomech 2005;20:892-9.

[27] Grood ES, Suntay WJ. A joint coordinate system for the clinical description of three-dimensional motions: application to the knee. J Biomech Eng 1983;105:136-44.

[28] Gage JR, DeLuca PA, Davis RB. Clinical gait analysis. A focus on interpretation. Hartford, Connecticut, USA: Connecticut Childrens Medical Center; 1997.

[29] Perry J. Gait analysis. Thorofare, NJ: SLACK Inc.; 1992.

[30] Stebbins J, Theologis T. Comparative motion of the foot during walking for men, women and children. Gait Posture 2009;30S:S1-53.

[31] Michaud TC. Foot orthoses and other forms of conservative foot care. Baltimore: Williams \& Wilkins; 1993. pp. 1-249.

[32] Kirtley C. Clinical gait analysis: theory and practice. Edinburgh: Churchill Livingstone; 2006. pp. 83-94.

[33] Tranberg R, Karlsson D. The relative skin movement of the foot: a 2D roentgen photogrammetric study. Clin Biomech 1998;13:71-6.

[34] Cappozzo A, Catani F, Leardini A, Benedetti MG, Della CU. Position and orientation in space of bones during movement: experimental artefacts. Clin Biomech 1996;11:90-100. 